This document is the Accepted Manuscript version of a Published Work that appeared in final form in J. Chem. Phys. 144, 154311 (2016), copyright $(\odot$ American Institute of Physics after peer review and technical editing by the publisher. To access the final edited and published work see

http://aip.scitation.org/doi/10.1063/1.4945780 


\title{
On the applicability of one- and many-electron quantum chemistry models for hydrated electron clusters.
}

\author{
László Turi ${ }^{1}$ \\ Eötvös Loránd University, Department of Physical Chemistry, Budapest 112, P. O. Box \\ 32, H-1518, Hungary
}

We evaluate the applicability of a hierarchy of quantum models in characterizing the binding energy of excess electrons to water clusters. In particular, we calculate the vertical detachment energy of an excess electron from water cluster anions with methods that include one-electron pseudopotential calculations, density functional theory (DFT) based calculations, and ab initio quantum chemistry using MP2 and eom-EA-CCSD levels of theory. The examined clusters range from the smallest cluster size $(n=2)$ up to nearly nanosize clusters with $n=1000$ molecules. The examined cluster configurations are extracted from mixed quantum-classical molecular dynamics trajectories of cluster anions with $n=1000$ water molecules using two different one-electron pseudopotenial models. We find that while MP2 calculations with large diffuse basis set provide a reasonable description for the hydrated electron system, DFT methods should be used with

\footnotetext{
${ }^{1}$ E-mail: turi@chem.elte.hu, fax: (36)-1-372-2592.
} 
precaution and only after careful benchmarking. Strictly tested one-electron psudopotentials can still be considered as reasonable alternatives to DFT methods, especially in large systems. The results of quantum chemistry calculations performed on configurations that represent possible excess electron binding motifs in the clusters appear to be consistent with the results using a cavity structure preferring one-electron pseudopotential for the hydrated electron, while they are in sharp disagreement with the structural predictions of a non-cavity model. 


\section{Introduction}

Negatively charged water clusters form a class of species with a set of challenging physical properties. ${ }^{1}$ The smallest water cluster that can bind an electron is the water dimer. ${ }^{2}$ Increasing the cluster size leads to an increasing stability of the excess electron and an increasing complexity of the cluster anion potential energy surface. Experimentally, this complexity is manifest in the variation of the vertical detachment energy (VDE), the energy needed to remove an electron from the clusters vertically, with cluster size., ${ }^{3,4,5,6,7}$ The VDE-size diagrams exhibit various systematic patterns that may indicate common structural motifs within the groups. Based on early ${ }^{8}$ and subsequent improved one-electron model quantum simulations, ${ }^{9,10}$ and an allelectron density functional theory based molecular dynamics study, ${ }^{11}$ tentative assignments of the different anionic groups have been proposed. Unfortunately, these theoretical predictions do not necessarily match the experimentally inferred classifications. ${ }^{3,4}$ In addition, recently a non-cavity structural model has been proposed based on a one-electron quantum model and simulation, ${ }^{12,13}$ questioning the traditionally accepted cavity structure of the hydrated electron. ${ }^{14}$ In summary, it is a challenging theoretical problem to settle these two conceptually important structural/energetic issues (i.e. surface states vs. interior states, and cavity vs. non-cavity structures).

The main problem is that the one-electron theoretical models $s^{15,16,17,18,19,20,21}$ that have been used to simulate and characterize the hydrated electron contain various approximations. These approximations, however, may be inherently reflected on the simulated structural and other physical properties. ${ }^{1}$ As many-electron calculations have become routinely available, the theoretical treatments of the hydrated electron system, both static $22,23,24$ and dynamic, $11,25,26,27,28,29,30,31$ shifted to density functional theory (DFT) based approaches. The limitations of 
the DFT methods, most importantly the choice of the exchange-correlation functional, are wellknown and documented for loosely-bound electrons. ${ }^{32}$ Clearly, quantum mechanical calculations at very high level of theory, and at very large basis set would be needed to address the problematic issues more reliably. Such treatments are, however, still very expensive. One possible route to improve the reliability of the hydrated electron theoretical results is to perform static benchmarking calculations and evaluate the available, computationally feasible methods in comparison with these high accuracy computations on relatively small systems. The evaluated methods then can be employed in large scale simulations and the predicted properties can be critically assessed in view of the benchmarking performance. The critical analysis of the performance of available, routinely employed theoretical models is one of the aims of the present study.

Although benchmarking calculations of the excess electron binding energies are available in the literature for water cluster anions, ${ }^{23,24,26,33}$ these studies usually focus on analyzing a limited set of configurations of small clusters. The latest, most comprehensive study by Vysotskiy et al. concludes that the equations-of-motion electronaffinity coupled-cluster-singlesdoubles (eom-EA-CCSD and eom-EA-CCSD(2)) methods ${ }^{34}$ with large diffuse basis sets are able to capture the VDE in both the weakly and strongly bound excess electron cases for water cluster anions in selected $\left(\mathrm{H}_{2} \mathrm{O}\right)_{\mathrm{n}}^{-}$cluster configurations with $n=4-24$ monomers. ${ }^{33}$ We start our analysis on the conclusions of this study. However, instead of selecting more or less arbitrary water cluster configurations, in most cases optimized anionic or neutral clusters, here we propose a different approach. We generate a large set of cluster configurations of various size using mixed one-electron quantum-classical molecular dynamics (QCMD) simulations, and then analyze the binding energies of these configurations using a hierarchy of computational methods. The results 
of one-electron based molecular dynamics simulations may strongly depend (both in energy, structure, spectroscopy and dynamics) on the applied electron-water pseudopotential. ${ }^{9,10,12,35}$ In the context of the present benchmarking procedure, configurations generated using different oneelectron models will serve as test cases for which one can directly evaluate and compare relevant physical properties with the results of more sophisticated quantum mechanical methods. In particular, we produce cluster configurations performing extensive molecular dynamics (MD) simulations using two different pseudopotentials, the Turi-Borgis (TB)19 and the Larsen-GloverSchwartz (LGS) $)^{12}$ potential. The TB model predicts a cavity structure, ${ }^{19}$ while the LGS model simulates a non-cavity type excess electron distribution in bulk hydrated electron simulations. ${ }^{12}$ Furthermore, the TB model simulates at least two types of cluster configurations. ${ }^{9,36,37}$ In the first isomer type, the electron is localized in the interior of the cluster in a cavity (interior state clusters), while the second isomer structure stabilizes the excess electron outside the cluster, on its surface (surface state clusters). TB and LGS molecular dynamics simulations on clusters thus produce configurations that can support surface state excess electrons, cavity type interior state electrons, and non-cavity type interior state electrons. ${ }^{35}$ These cluster structures will form representative samples for the quantum mechanical binding energy calculations and the following analysis. In this respect, this series of calculations provides a comprehensive analysis of the hydrated electron system. The present calculations can also offer hints as which type of oneelectron MD generated cluster anion configurations has physical properties that are more consistent with the many-electron calculations.

Here we mention that the Jordan group ${ }^{21,38}$ and the Herbert group also compared VDE of water cluster anions computed using pseudopotential models to ab initio calculations. ${ }^{20,39}$ Their structures were, however, to the best of our knowledge, not taken from MD simulations, the main 
point of the present analysis. The MD generated configurations can also be compared, at least qualitatively, with the electron binding motifs found experimentally in vibrational predissociation spectra by the Johnson group. ${ }^{40,41}$ They identified two different classes for the electron binding sites in $\left(\mathrm{H}_{2} \mathrm{O}\right)_{6}^{-}$clusters, one with a double hydrogen-bonding acceptor (AA) water molecule pointing in the excess electron distribution with two hydrogen atoms, and the other with several, hydrogen-bond acceptor-donor (AD) water molecules with oriented dangling hydrogen atoms toward the electron. Of the two isomers, the one with the AA type water molecule binds the electron more strongly.

A different (and in some sense, more fundamental) aspect, a comparison of the electronwater molecule pseudopotentials in predicting experimental physical properties of hydrated electron clusters, has also been published recently. ${ }^{35}$ In that work, we referred to preliminary quantum chemical VDE calculations that appear to be fully consistent with the other findings of the paper. ${ }^{35}$ The present work provides the full quantum chemical analysis of the problem completing the comparison of pseudopotentials and giving a solid theoretical ground for the previous conclusions.

The structure of the paper will be as follows. In Sec. II, we briefly describe the main features of the QCMD techniques used to generate the configuration sets, and the quantum mechanical computational methods that are employed in the present study. Sec III collects the results of the energy calculations followed by a systematic analysis and comparison of the results. Sec IV provides a discussion, and concludes the paper.

\section{Computational details}


First, we performed one-electron QCMD simulations on water cluster anions containing 1000 water molecules. The applied QCMD simulation technique has been developed by Rossky et $a l^{42}$ The details of the simulations are identical to those described in our previous papers. $^{9,19,35,37,43}$ Since the application of the QCMD technique is well documented (see references in Ref 1), we mention shortly that in one-electron hydrated electron QCMD simulations the Schrödinger equation of the excess electron is solved in the field of classical water molecules, in the present case a three-site, simple point charge, flexible model $(\mathrm{SPC}+\mathrm{flex}){ }^{44}$ The critical part of the present method is the choice of the electron-water molecule potential. Several pseudopotential models have been proposed in the literature..$^{15,16,17,18,19,20,12}$ Of these models, we use the $\mathrm{TB}^{19}$ and the $\mathrm{LGS}^{12}$ potentials to generate equilibrated trajectories at $\sim 200 \mathrm{~K}$. The main reason for selecting these two potentials is that, while both potentials employ the same non-polarizable water-water potential, and both are developed using the same implementation ${ }^{45}$ of the static-exchange pseudopotential theory, ${ }^{46}$ they produce a significantly different solvation structure for the excess electron. ${ }^{35}$

Here we note that pseudopotentials, taking solvent polarization explicitly into account, have also been developed. ${ }^{18,20,21}$ These polarizable models are of higher theoretical sophistication, than the LGS or TB models that include polarization in an average way. In the present paper, however, the TB and LGS models serve to generate characteristic (and realistic) water cluster anion configurations to be analyzed. These potentials suffice for this purpose. Furthermore, the use of rigid water monomers in most of the polarizable models ${ }^{18,21}$ (with the exception of the Jacobson-Herbert potential $)^{20}$ does not allow application of these potentials to the present nonrigid structures. Therefore, although we readily acknowledge the merits of the polarizable models 
(especially those $\mathrm{e}^{20,21}$ that outperform simple DFT or small basis MP2 methods), we omit them from the present comparison.

We generated $100 \mathrm{ps}$ long equilibrium trajectories at $200 \mathrm{~K}$ using both potentials with a time step of 1 fs. In addition, we produced separate trajectories with the TB potential for surface state clusters and interior state clusters, ${ }^{9,36}$ as well. We chose $100-100$ configurations of each of the three trajectories with 1 ps time segments between the selected individual configurations. For further analysis, in each of the configurations, we determined the center of mass of the hydrated electron, computed with the pseudopotential pertinent to the given trajectory, and produced clusters of gradually increasing size, consisting of $n$ water molecules $(n=2-1000)$ that are nearest to the center of mass of the electron. For these cluster arrangements we performed various level quantum mechanical calculations. We computed the relevant energies for 100 configurations in the $n=2-12$ size range, 20 configurations with $n=16$ and 20 , and 10 configurations with $n=24$ using many-electron methods. All the data (geometries and energies) can be found in the Supplementary Material (with a very few problematic (convergence issues) cases). The VDE was computed for 100-100 configurations with one-electron pseudopotentials for all examined cluster sizes. The clusters are identified by the pseudopotential that was used to generate the parent trajectory and by the number of water molecules carved out of the $n=1000$ original cluster. The investigated structures will be referred to as TB generated interior or surface structures, or LGS generated non-cavity structures. We note that these names refer only to the electron binding motifs of the parent $n=1000$ clusters, and they do not necessarily describe the interior or surface character of the computed excess electron distribution in the smaller investigated structures. 
The three structure sets are distinctly different. We show three characteristic hexamer examples of the investigated structures in Figure 1. In a typical TB interior configuration, the interior cavity is clearly visible. Five of the six monomers that form the cavity in Figure 1 point with one of their hydrogen atoms in the interior of the cavity. The second hydrogen atoms of the water molecules participate in hydrogen bonds with the water molecules of the second solvation shell (not shown). The LGS generated interior structures are significantly different from the TB interior configurations at least in three respects. 1) The LGS structures are more compact around the localization site of the excess electron than the TB configurations. 2) No localization cavity is observable in most of the LGS structures. 3) The hydrogen-bonded network around the localization site does not differ discernibly from that of pure water. The excess electrons in the TB surface configurations (bottom of Figure 1) are localized around dangling hydrogen atoms on the cluster surface. The typical signature of these clusters, (at least) one AA water molecule that participates in two hydrogen bonds as an acceptor and has two dangling hydrogen atoms oriented toward the electron, can be easily identified in Figure 1.

The main quantity we focus on is the vertical detachment energy of the excess electron, the difference of the energy of the anionic and the neutral species in the same geometry. The methods of the VDE calculations include a) one-electron pseudopotential quantum mechanical calculations using the TB and LGS pseudopotentials, b) various density functional theory (DFT) methods, c) second order Moller-Plesset methods, and d) eom-EA-CCSD calculations. We note that in the non-polarizable TB and LGS models the VDE is equivalent to the ground-state eigenvalue of the excess electron. The one-electron pseudopotential calculations are performed on each type of configurations including those generated by the other model. We also carried out DFT calculations using four different exchange-correlation functionals that were used previously 
in hydrated electron simulations, the gradient corrected BLYP functional, ${ }^{47}$ the long range corrected BLYP functional (LC-BLYP), ${ }^{47,48}$ the hybrid BHandHLYP functional, ${ }^{49}$ and the PBE functional. ${ }^{50}$ Of the standard quantum chemistry methods that include correlation energy, we employed the simplest MP2 perturbation method.$^{51}$ For comparison we also applied the eom-EACCSD method ${ }^{34}$ that has been shown recently to be a reliable, although expensive, method to calculate precise vertical detachment energies for small water cluster anions. ${ }^{33}$ The choice of the basis set is a critical issue in hydrated electron cluster simulations due to the diffuse nature of the excess electron. We used two basis sets, both developed by Herbert and Head-Gordon, the 6$31(1+3+) \mathrm{G}^{*}$ and the aug3-cc-pVDZ sets, both containing very diffuse extra functions in addition to the standard sets. ${ }^{23}$ Although these two basis sets have been demonstrated to provide relatively good results in comparison with larger, more extensive, for example, triple-zeta quality sets, ${ }^{23,24}$ we further illustrate this feature in more detail below. The eom-EA-CCSD/aug3-cc-pVDZ calculations were performed with the CFOUR program package, ${ }^{52}$ while all other quantum chemistry calculations were executed using the Gaussian09 program suite. ${ }^{53}$

\section{Results}

The first task is to test the applied basis sets of the quantum chemistry calculations. Although the performance of the $6-31(1+3+) \mathrm{G}^{*}$ and the aug3-cc-pVDZ sets has been demonstrated in previous hydrated electron calculations, ${ }^{23,24}$ we decided to perform an additional test employing these basis sets. We carried out the analysis on hexamer configurations, and calculated the VDE with the above two basis sets. The calculations were performed on $20-20$ configurations of all three configuration sets produced in the QCMD simulations driven by the LGS, and the TB pseudopotentials (LGS, TB interior and TB surface sets). Typical structures are 
shown in Figure 1. We emphasize that these structures are carved out of larger, $n=1000$ clusters and contain the molecules that are nearest to the computed center-of-mass of the excess electron. The $n=4$ and $n=6$ clusters include those molecules that are in direct contact with the excess electron distribution at the localization site (first solvation shell). Increasing the cluster size adds most likely hydrogen-bonding water molecules to the cluster that stabilize the molecules of the first solvation shell (second, third, etc. solvation shells).

We carried out the calculations with MP2, BHandHLYP and LC-BLYP methods. To check reasonable convergence of the basis, we performed calculations using the standard aug-ccpVDZ set, the aug3-cc-pVTZ, and the aug4-cc-pVDZ sets. The calculated average VDE values are collected in Table I. To provide a statistically more informative analysis on the performance of the basis sets, we also computed the average difference between the VDE values computed with a smaller basis set and a larger basis set, defined as follows:

$$
\sigma(A, B)=\sqrt{\frac{\sum_{i}\left[(V D E(i, A)-V D E(i, B)]^{2}\right.}{n}}
$$

In Eq (1), the sum runs over the investigated configurations with index $i, n$ is the number of configurations, and $A$ and $B$ denote the basis sets with $A=6-31(1+3+) \mathrm{G}^{*}$, aug-cc-pVDZ, aug3-ccpVDZ, and $B=$ aug4-cc-pVDZ, aug3-cc-pVTZ.

As a first observation, one notices that all five basis sets provide qualitatively similar results within the configuration groups, except the aug-cc-pVDZ basis for the LGS geometry. The general trend within this configuration set, small VDE values for the LGS geometries, suggest that the excess electron is weakly bound and diffuse. Clearly, the diffuse functions of the aug-cc-pVDZ set are unable to capture this character, and this causes the large negative VDE values in Table I. Additional diffuse functions stabilize the anion leading to small, but positive 
VDE. We further analyze and discuss the small VDE values of the LGS set below. Due to the failure of the aug-cc-pVDZ basis within the LGS configuration set, although, there is no major difference between the VDE values of the aug-cc-pVDZ and the larger basis sets for the TB structures, we exclude this basis set from further considerations. Next, we inspect whether the two extra sets of diffuse functions in the aug3-cc-pVDZ are sufficient to reach reasonable agreement with aug3-cc-pVTZ and the aug4-cc-pVDZ sets. The VDE values rapidly converge (within 10-20 meV) for all type of configurations (Table 1) suggesting that the aug3-cc-pVDZ basis is a reasonable choice to characterize the VDE values of water cluster anions. The average VDE differences of the aug3-cc-pVDZ basis relative to the larger aug4-cc-pVDZ and aug3-ccpVTZ sets underline these observations on the basis set convergence. Increasing the number of diffuse sets from aug3 to aug4 or changing from VDZ to VTZ causes only moderate effect, crudely less than an average of $\sim 20 \mathrm{meV}$ difference in the individual VDE values. We note that although this difference seems quite small, it becomes significant in relative terms for the weakly binding LGS structures. One can also see that the use of additional diffuse shells influences the VDE of these delocalized, weakly bound excess electron cases more strongly than the use of additional valence shells. Clearly, accurate description of the low VDE cases would require significantly larger basis sets than those employed here. The situation is reversed for the strongly bound interior cavity and surface states, where additional valance functions have greater effect on the anions' stability than the extra diffuse functions. Nevertheless, we argue that the crudely 20 meV accuracy of the aug3-cc-pVDZ VDE values in all three examined groups of configurations is sufficient for the general purposes of this paper. Last, we examine the performance of a significantly cheaper, split-shell basis augmented with diffuse functions, the $6-31(1+3+) \mathrm{G}^{*}$ basis, proposed originally by Herbert and Head-Gordon. ${ }^{23}$ This basis still relatively nicely fares 
compared to the more sophisticated augn-cc-pVXZ $(n=3,4 ; \mathrm{X}=\mathrm{D}, \mathrm{T})$ basis sets. To illustrate this point we show a correlation diagram for the VDE, computed with $6-31(1+3+) \mathrm{G}^{*}$ vs. aug3-ccpVDZ for the examined hexamer structures (Figure 2). The relation is apparently linear, with a slope of 1.137 and an intercept of 0.005 for the fitted straight line. This indicates that although the $6-31(1+3+) \mathrm{G}^{*}$ overestimates the VDE (in some cases by $\sim 15 \%$ for the strongly bound clusters), it nicely follows the aug3-cc-pVDZ general tendencies. Despite this VDE overestimation, we shall use the $6-31(1+3+) \mathrm{G}^{*}$ basis in our more extensive calculations later in the paper, while the aug3-cc-pVDZ set will be employed for more accurate benchmarking purposes.

In the next step, we inspect the employed computational techniques and examine how the VDE values obtained with the cheaper MP2 calculations compare with those using the high-level eom-EA-CCSD/aug3-cc-pVDZ method. For this, we computed the VDE for selected tetramer and hexamer configurations for each type of structures with the eom-EA-CCSD/aug3-cc-pVDZ method. The eom-EA-CCSD/aug3-cc-pVDZ calculations were performed with the CFOUR program package. The bottleneck of these calculations was the extremely slow SCF convergence of the anions in the CFOUR package, especially for the LGS configurations. For this reason, we increased the SCF convergence to $10^{-6}$ from the default $10^{-7}$ for the LGS tetramers. The TB configurations were computed with the default value. Tests performed on the converged TB structures showed that this technical step has no effect on the VDE values. All in all, for the tetramers we computed the eom-EA-CCSD/aug3-cc-pVDZ VDE for ten TB surface configurations, ten TB interior configurations and nine LGS configurations (with the looser convergence criterion). We also calculated the eom-EA-CCSD/aug3-cc-pVDZ VDE for six TB surface and five TB interior hexamer configurations. Our attempts to reach SCF convergence for 
all the LGS hexamers failed using the CFOUR package. We suspect that the reason for these convergence difficulties may be of technical nature. About the stability of the anionic species we note that all generated anionic tetramer and hexamer TB structures are predicted to be bound, while of the nine converged cases of the anionic LGS tetramer configurations only four proved to be stable with respect to their VDE using the eom-EA-CCSD/aug3-cc-pVDZ method. Now we compare the computed eom-EA-CCSD/aug3-cc-pVDZ VDE values to MP2/aug3-cc-pVDZ and MP2/6-31(1+3+) $\mathrm{G}^{*}$ results, considering only the positive VDE cases. Figure 3 shows the correlation between the eom-EA-CCSD results and MP2 calculations. The MP2 tendencies essentially capture the eom-EA-CCSD/aug3-cc-pVDZ behavior. Nevertheless, we observe that a) the MP2 numbers are too weak by $\sim 70-80 \mathrm{meV}$ in the lowest VDE regime (indicated by the negative intercept of the correlation line), and b) MP2 overestimates the VDE values above $\sim 0.5$ $\mathrm{eV}\left(\mathrm{MP} 2 / 6-31(1+3+) \mathrm{G}^{*}\right)$ and $\sim 1 \mathrm{eV}(\mathrm{MP} 2 / \mathrm{aug} 3-\mathrm{cc}-\mathrm{pVDZ})$. This is the result of overestimated slopes of 1.18 and 1.06 with the 6-31(1+3+)G* and aug3-cc-pVDZ basis sets, respectively.

Now we begin our analysis on larger cluster configurations using a variety of computational methods. The many-electron calculations are performed with the $6-31(1+3+) G^{*}$ basis set, and we compare these VDE values to the MP2/6-31(1+3+) $\mathrm{G}^{*}$ results. Here we would like to point out that, although $6-31(1+3+) \mathrm{G}^{*}$ is a relatively small basis set, it describes the general VDE tendencies surprisingly well. Nevertheless, it is known that the reasonable behavior of the MP2/6-31(1+3+) G* method is the result of fortuitous cancellation of errors. ${ }^{23,32}$ Part of the problems originates from the fact that this basis was optimized for structures that bind the excess electron more strongly than $\sim 0.5 \mathrm{eV} .^{23}$ All in all, despite the success of the $6-31(1+3+) \mathrm{G}^{*}$ basis set one has to be aware of its deficiencies. For this reason, we correct the MP2/6-31(1+3+)G* VDE values empirically for their underestimation in the low VDE regime, and for their 
overestimation for the more strongly bound species using a linear scaling to the eom-EACCSD/aug3-cc-pVDZ data for tetramers and hexamers (see the correlation line of Figure 3). In the following we examine the three configuration groups, the TB interior, the TB surface and the LGS configuration sets, separately.

The computed VDE values for the TB potential generated configurations where the excess electron is stabilized in an interior state in QCMD simulations are shown in Figure 4. These configurations contain a preformed cavity within the cluster with hydrogen atoms orienting preferably toward the cavity's center. All examined methods with the exception of the TB potential overestimate the corrected MP2 values. The corrected MP2 VDE value at the largest examined cluster size $(n=24)$ is $1330 \mathrm{meV}$, while one-electron pseudopotentials give $1050 \mathrm{meV}$ (TB) and $2020 \mathrm{meV}$ (LGS). The large size behavior of the TB model $(3.7 \mathrm{eV}$ VDE for the $n=$ 1000 cluster) compares favorably to the direct experimental measurement of the VDE for the bulk hydrated electron by the Neumark group $(3.6 \mathrm{eV}),{ }^{54}$ while the LGS potential predicts a larger value of $5.0 \mathrm{eV}$. The VDE values of the DFT methods are significantly overestimated relative to the corrected MP2 VDE results that we use as our benchmark data. The PBE and BLYP methods predict similar values, significantly overestimating both the corrected and the uncorrected MP2 VDE results ( 50\%), while the LC-BLYP and BHandHLYP functionals compute values that are more reasonably approximate the MP2 values $(\sim 30 \%)$. Here we note that since the use of the $6-31(1+3+) G^{*}$ basis sets results in larger VDE relative to larger diffuse sets (see Figure 2), we expect, that the overestimation tendency would be somewhat diminished in larger basis set DFT computations.

The VDE values computed on the TB potential generated surface state configurations are collected in Figure 5. We remind that surface state configurations have large dipole moments and 
a slight dent on the surface with free, dangling hydrogen atoms. ${ }^{36,55}$ The general tendencies of the computed VDE values are similar to the interior case, but here we emphasize that both oneelectron methods give a fair estimate of the MP2 values. While LGS results compare favorably to the uncorrected MP2 values (1670 meV vs $1550 \mathrm{meV}$ for $n=24)$, the TB numbers show good agreement with the corrected MP2 results (1230 meV vs $1370 \mathrm{meV}$ for $n=24)$. The overestimation of the uncorrected MP2 values by the DFT methods is more moderate for the surface state configurations, but still significant for the PBE and BLYP methods ( 50\%), while it is less dramatic $(\sim 30 \%)$ with the LC-BLYP and BHandHLYP functionals.

The third test group of water cluster anions are formed by those configurations that were generated in QCMD simulations using the LGS pseudopotential. In these configurations, no preformed cavity or surface binding site for the excess electron are present. In fact, the LGS model predicts that the electron localizes in a region of locally enhanced water density within the cluster. ${ }^{35}$ The computed VDE data on this set of configurations are collected in Figure 6. The first, most obvious observation, that were already seen in Table I, is that all methods except LGS predict significantly smaller VDE than for surface or interior cavity configurations. The MP2 uncorrected and corrected values are around $100 \mathrm{meV}$ at $n=24$, suggesting that the excess electron is very weakly bound to the clusters. The one-electron TB potential only slightly underestimates MP2. The LGS potential, however, gives VDE values that shoot up very quickly to $3.5 \mathrm{eV}$ at $n=24$. The LGS numbers are fully incompatible with all other many-electron calculations, and appear to be unphysical. Of the DFT methods, PBE and BLYP methods predict relatively strong interactions up to $\sim 1 \mathrm{eV}$ at $n=24$, which again seem to be way too strong. For the non-cavity configurations BHandHLYP and LC-BLYP follow the MP2 tendency quite reasonably. 
For a comparison, the large cluster size behaviors of the one-electron LGS and TB models are illustrated in Figure 7. As we observed LGS model predicts stronger electron attachment to water clusters than the TB potential in each of the three groups of configurations including the TB generated cavity type interior and surface state configurations, as well. Both simple models predict that the interior cavity structure is more strongly binding than the surface state configurations, the difference at the $n=1000$ cluster size is $\sim 0.6 \mathrm{eV}$. Interestingly, the corrected MP2 numbers are practically identical for surface state and interior state configurations in the examined $n=2-24$ size range. Significant differences are apparent in the VDE values of the LGS generated non-cavity interior structures. While the TB model, similarly to the trends for most many-electron calculations, computes only slight stabilization of the excess electron on these clusters, the LGS model predicts a huge binding, $\sim 7 \mathrm{eV}$ at the $n=1000$ cluster size. This value is a factor 2 larger than the experimentally observed bulk VDE $(3.6 \mathrm{eV}),{ }^{54}$ the infinite size extrapolated limit of the cluster VDE. One may note that the experimental bulk VDE is in more sensible agreement with the TB model for interior cavity type configurations.

At the end of the section we discuss the larger cluster size behavior of the MP2/6$31(1+3+) \mathrm{G}^{*}$ computed VDE data of the TB interior state and surface state configurations. First, we note that the VDE is practically identical for the TB interior and surface state configurations as computed with MP2/6-31 $(1+3+) \mathrm{G}^{*}$ method up to $n=24$. To further extend the examined size range, we performed VDE calculations on additional 10-10 selected configurations of the TB generated interior state and surface state configuration sets with $n=36$ water molecules. We corrected the MP2 data according to our previous empirical scaling based on Figure 3. The average VDE's are $1.57 \mathrm{eV}$ and $1.74 \mathrm{eV}$ for $n=36$ interior and surface state configurations, respectively. Interestingly, the surface state VDE becomes in average noticeably greater than that 
for interior states. This tendency parallels the size dependence of the TB calculated VDE values for $n<300$ cluster sizes. This trend is also clear in Figure 8 which shows the correlation between the TB data and the corrected MP2 values. Furthermore, the correlation, although it is reasonably linear, indicates that the TB results underestimate the MP2 predicted VDE values. The TB pseudopotential predicts $4.4 \mathrm{eV}$ for interior states and $3.9 \mathrm{eV}$ for surface states in the infinite size limit of water cluster anions. ${ }^{36}$ These values are somewhat stronger than those extrapolated from cluster data $(3.3 \mathrm{eV})^{56}$ and those from direct bulk VDE measurements $(3.3-3.6 \mathrm{eV}) .^{54,57,58}$ The corresponding MP2 values, assuming the validity of the linear correlation of Figure 8 in the large cluster size limit, are $\sim 5 \mathrm{eV}$ and $\sim 4.5 \mathrm{eV}$. Thus, the MP2 estimate shifts the computed large size limit of the cluster VDE even farther from the experiment. A probable reason for this anomaly is that the TB model does not sample the MP2 configuration space of the anion quite correctly. According to a plausible assumption, MP2 prefers a somewhat larger cavity than the TB model. If that is the case, although larger MP2 cavities result in more stable structures for both the anionic and the neutral species, one may hypothesize that the stabilization for the neutral species in the anion's geometry with a larger cavity is greater than for the parent anion, leading overall to a decrease in the VDE values.

\section{Discussion and Conclusions}

We performed a series of comparative VDE calculations on anionic water configurations that were generated using one-electron QCMD simulations with two different electron-water molecule pseudopotentials. The configurations were selected from three possible types of water cluster arrangements that have been proposed to support the binding of an excess electron in water cluster anions. The configurations included a set of molecular arrangements that 
correspond to the traditional cavity type hydrated electron structure in the interior of the cluster, a recently proposed non-cavity type binding model in the interior, and a cluster isomer type with the electron attached to the cluster surface (surface-bound state).

We examined the performance of several quantum mechanical computational methods with respect to the vertical detachment energy of the excess electron from various size cluster anions. For the smallest cluster sizes (up to $n=6$ ) we found that MP2 calculations combined with reasonably diffuse basis set satisfactorily reproduce the tendencies of the most sophisticated method used in the present study, eom-EA-CCSD/aug3-cc-pVDZ. Based on the reasonable linear correlation between MP2/6-31(1+3+) $\mathrm{G}^{*}$ and eom-EA-CCSD/aug3-cc-pVDZ methods, we proposed that VDE calculations using more approximate quantum chemical methods (i.e. DFT and one-electron methods) will be compared to the empirically corrected MP2/6-31(1+3+) $\mathrm{G}^{*}$ results for larger cluster anions.

Based on the comparisons we find that all examined many-electron calculation methods predict strong binding of the excess electron to the cavity configurations (TB interior clusters) and to the configurations supporting surface bound states (TB surface clusters), while the VDE values become distinctly lower for the non-cavity configurations (generated by the LGS potential). In particular, the general tendencies of the VDE's among and within the three classes of water cluster anions are qualitatively similar for all examined DFT methods. The BLYP and the PBE functionals predict trends significantly overestimating the MP2 data (for example, by $>1$ $\mathrm{eV}$ for the $n=24$ clusters). These methods suggest relatively large binding energy even for the weakly binding non-cavity structures. Although the LC-BLYP and BHandHLYP methods represent considerable improvement over the previous two functionals, they still tend to overestimate the benchmark data by about $0.5 \mathrm{eV}$ for the largest examined cluster sizes for both 
the cavity type structures and the surface bound states, while predicting a smaller, $\sim 0.2 \mathrm{eV}$ overbinding for the weakly bound non-cavity cluster arrangements. Of the one-electron pseudopotentials, we observed that the VDE numbers computed with the TB potential consistently follow the MP2 tendencies. The TB numbers (for $n=24$ ) underestimate the corrected MP2 data by $0.2-0.3 \mathrm{eV}$ for the cavity structures and $0.1-0.2 \mathrm{eV}$ for surface bound states, the underestimation is even smaller for the non-cavity structures. The LGS method behaves similarly to the better performing DFT methods for both the cavity structures and the surface bound states. However, the LGS numbers for the non-cavity type structures reveal a serious mismatch relative to all other methods. While high level quantum chemistry calculations clearly indicate that these structures only very weakly bind the excess electron, we find nonrealistically large VDE values computed with the LGS pseudopotential. In fact, these structures are predicted by the LGS potential to be significantly more strongly bound for the excess electron than the TB cavity and surface state configurations. With this finding one can suspect that the LGS pseudopotential generates non-physical molecular configurations for water cluster anions and this observation points to the underlying problems with the LGS potential. These problems were suspected previously ${ }^{59,60,35}$ but this study clearly indicates anomalies associated with the LGS potential.

The present study also provides an opportunity to estimate bulk VDE values. The TuriBorgis pseudopotential predicts $4.4 \mathrm{eV}$ for interior states and $3.9 \mathrm{eV}$ for surface states in the large cluster limit of water cluster anions. ${ }^{36}$ These values are somewhat stronger than from direct bulk VDE measurements of 3.3-3.6 eV. We also find that the MP2 predicted VDE values on large TB generated configurations are roughly $10 \%$ larger, further increasing the gap between the calculated and the measured VDE values. This suggests that the sampling of the configuration 
space by the TB model may not be completely satisfactory. Nevertheless, this model still provides valuable semi-quantitative information on the physical behavior of negatively charged water cluster anions. On the other hand, significant difference appears between commonly used DFT methods and MP2 calculations. In particular, we conclude that while DFT methods should be used with certain precautions for the hydrated electron system, carefully parameterized oneelectron pseudopotential models still offer reasonable and viable alternative to the application of more expensive all-electron approaches, especially in large systems.

\section{Acknowledgments}

This work was supported by a research grant to LT from the National Research Fund of Hungary (OTKA, K104237). 


\section{Figure Captions}

Figure 1. Typical TB interior (top), LGS interior (middle) and TB surface structures (bottom) containing six molecules that are nearest to the center-of-mass of the excess electron in the corresponding MD generated $n=1000$ clusters.

Figure 2. Correlation of the vertical detachment energies of $\left(\mathrm{H}_{2} \mathrm{O}\right)_{6}^{-}$water cluster anions computed with MP2/6-31(1+3+)G* vs. MP2/aug3-cc-pVDZ. Red: LGS non-cavity configurations; blue: TB interior cavity configurations; green: TB surface state configurations.

Figure 3. Comparison of vertical detachment energies of $\left(\mathrm{H}_{2} \mathrm{O}\right)_{4}^{-}$and $\left(\mathrm{H}_{2} \mathrm{O}\right)_{6}^{-}$water cluster anions computed with MP2/aug3-cc-pVDZ (green) and MP2/6-31(1+3+)G* (red) vs eom-EACCSD/aug3-cc-pVDZ.

Figure 4. VDE values of various size $\left(\mathrm{H}_{2} \mathrm{O}\right)_{n}^{-}$water cluster anions $(n=2-24)$ computed with selected quantum chemical methods on interior cavity configurations taken from TB potential generated QCMD trajectories. The DFT and MP2 calculations were performed using the 6$31(1+3+) \mathrm{G}^{*}$ basis set. The stars indicate the corrected MP2 values (MP2/corrected) based on the red correlation line of Figure 3.

Figure 5. VDE values of various size $\left(\mathrm{H}_{2} \mathrm{O}\right)_{n}^{-}$water cluster anions $(n=2-24)$ computed with selected quantum chemical methods on surface state configurations taken from TB potential generated QCMD trajectories. The DFT and MP2 calculations were performed using the 6$31(1+3+) \mathrm{G}^{*}$ basis set. The stars indicate the corrected MP2 values (MP2/corrected) based on the red correlation line of Figure 3. 
Figure 6. VDE values of various size $\left(\mathrm{H}_{2} \mathrm{O}\right)_{n}^{-}$water cluster anions $(n=2-24)$ computed with selected quantum chemical methods on non-cavity interior state configurations taken from LGS potential generated QCMD trajectories. The DFT and MP2 calculations were performed using the $6-31(1+3+) \mathrm{G}^{*}$ basis set. The stars indicate the corrected MP2 values (MP2/corrected) based on the red correlation line of Figure 3.

Figure 7. VDE values of various size $\left(\mathrm{H}_{2} \mathrm{O}\right)_{n}^{-}$water cluster anions $(n=2-1000)$ computed with one-electron LGS (blue) and TB (red) pseudopotential models. The calculations were performed on interior cavity configurations taken from QCMD trajectories with the TB potential ( $\mathbf{m})$, surface state configurations taken from QCMD trajectories with the TB potential $(\mathbf{\Delta})$, and non-cavity interior state configurations taken from QCMD trajectories with the LGS potential ( $\boldsymbol{\nabla})$.

Figure 8. Comparison of vertical detachment energies of TB model generated $\left(\mathrm{H}_{2} \mathrm{O}\right)_{36}^{-}$water cluster anions computed with the TB model vs corrected MP2/6-31 $(1+3+) \mathrm{G}^{*}$ values for interior (घ) and surface bound structures ( $\boldsymbol{\Delta})$. The dashed fitted line illustrates the correlation. 
Figure 1.

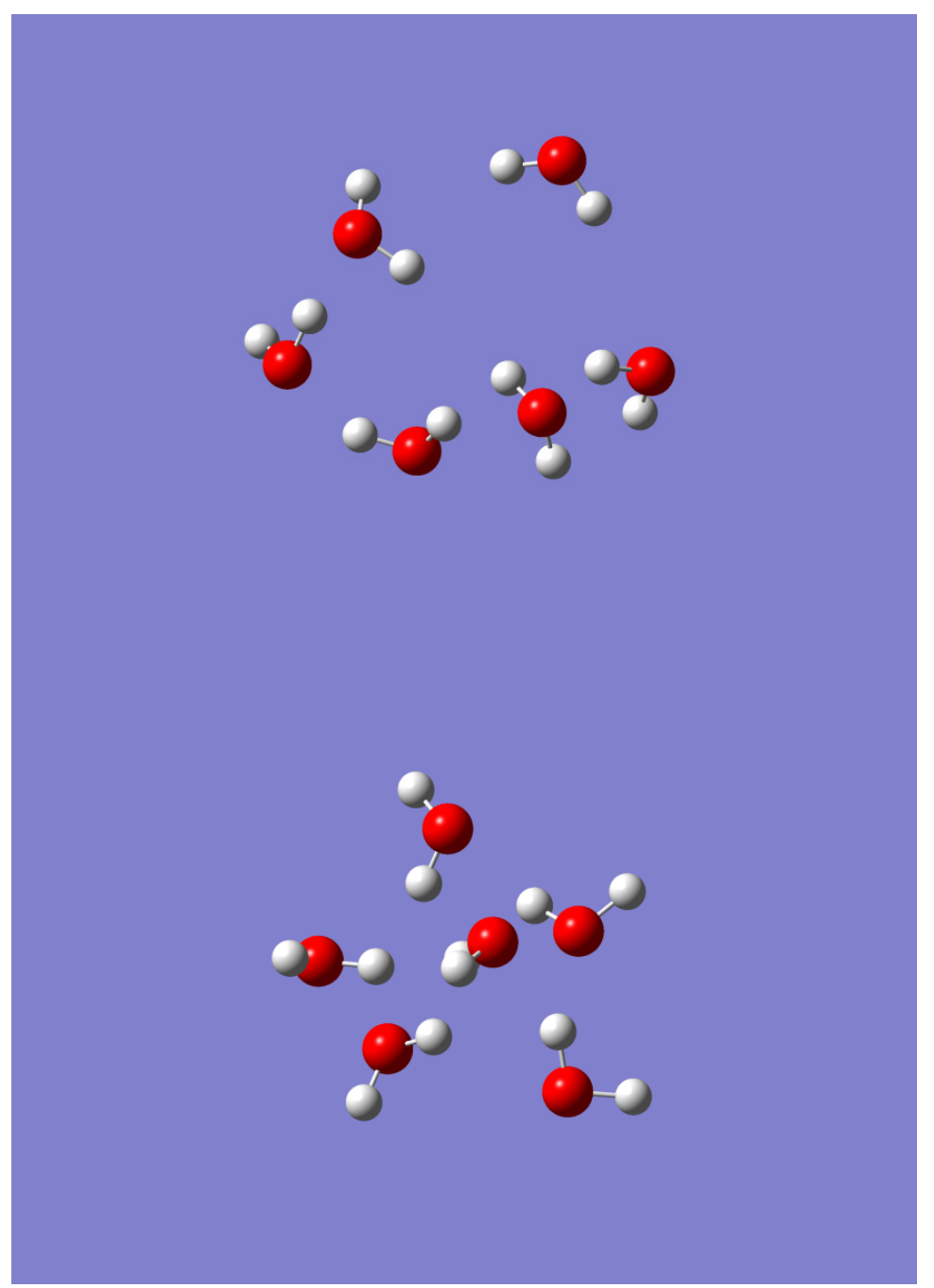




$$
\begin{aligned}
& 0^{2} \\
& \begin{array}{ll}
9 \\
0 \\
0 & 0
\end{array}
\end{aligned}
$$


Figure 2.

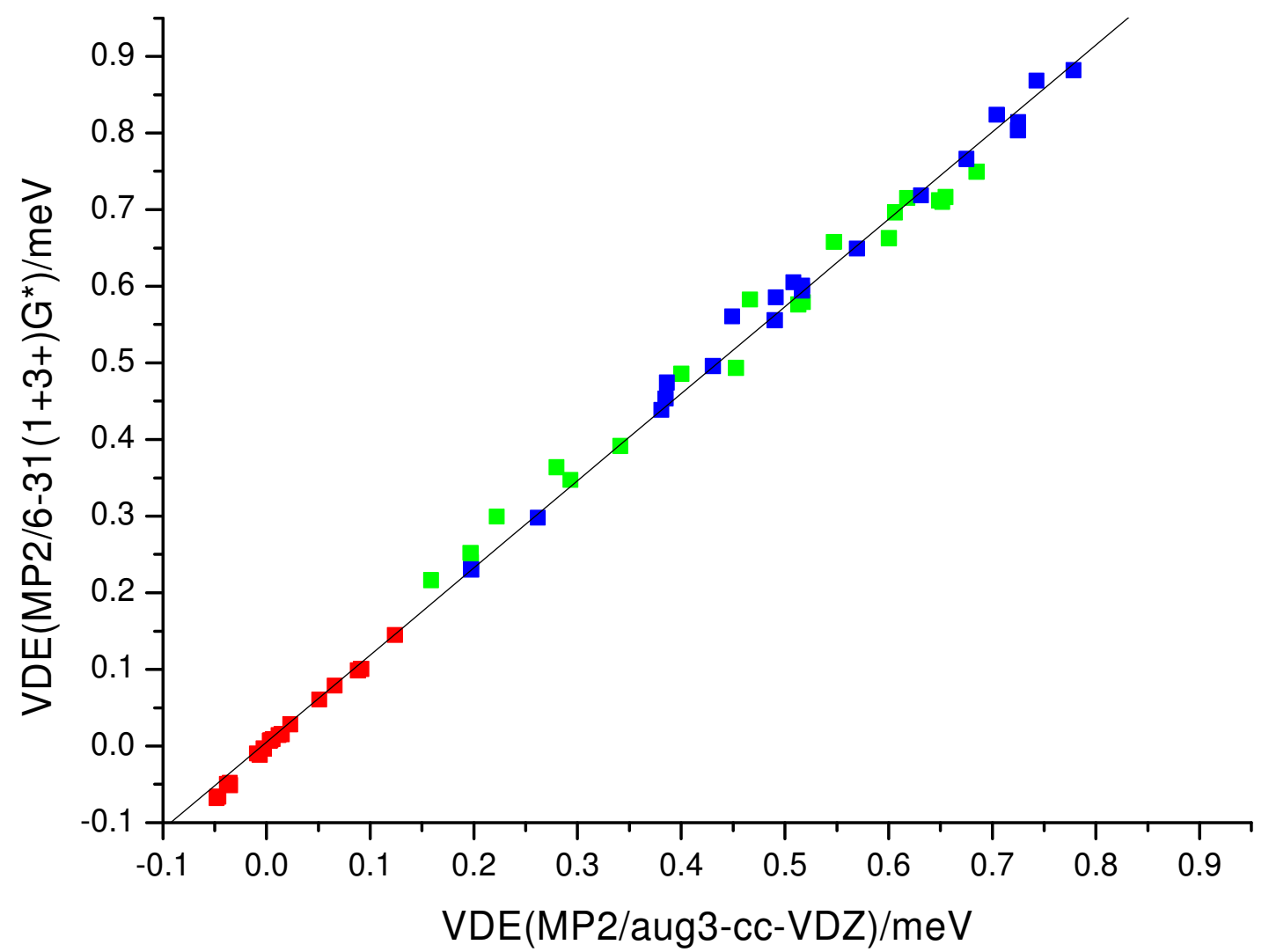


Figure 3.

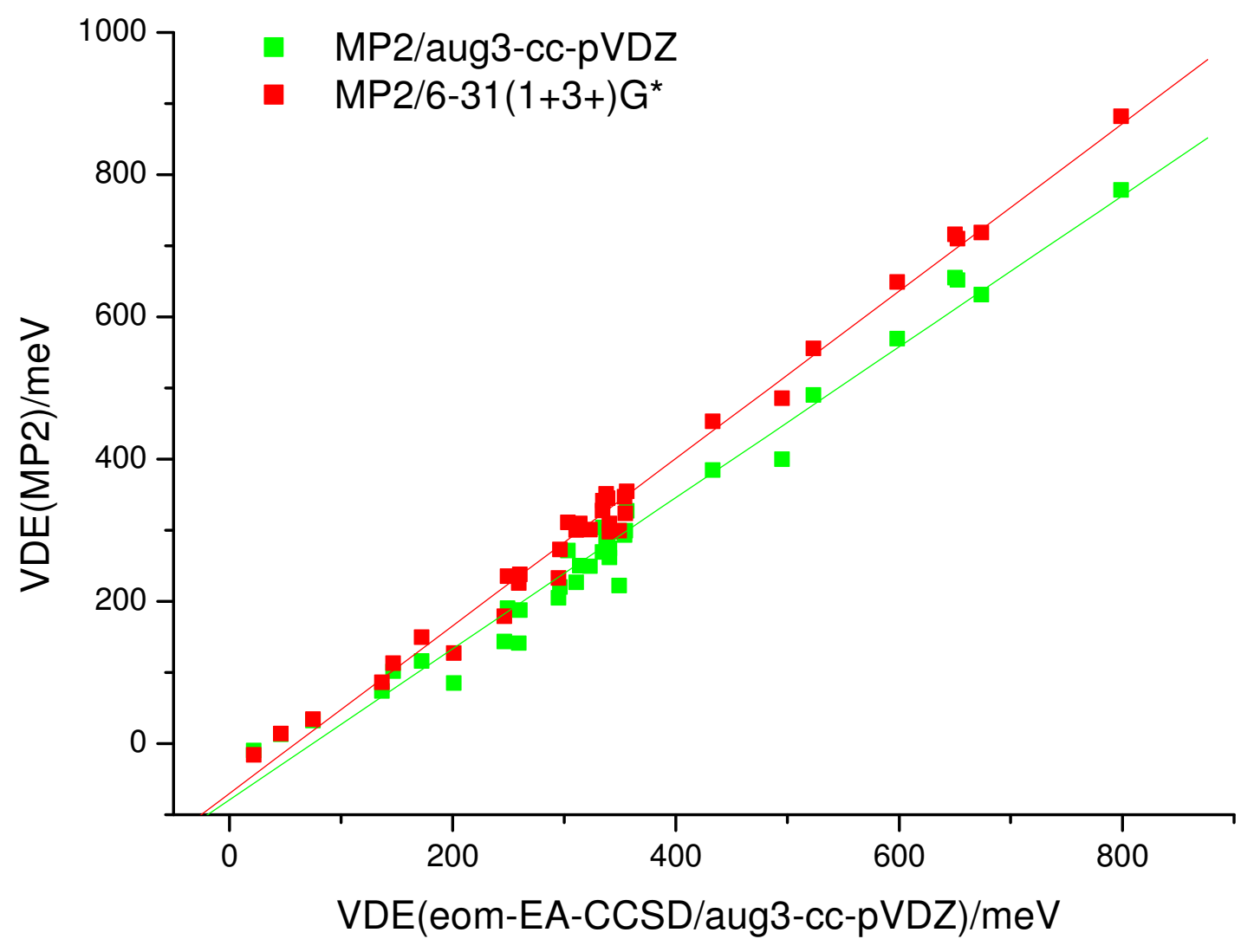


Figure 4.

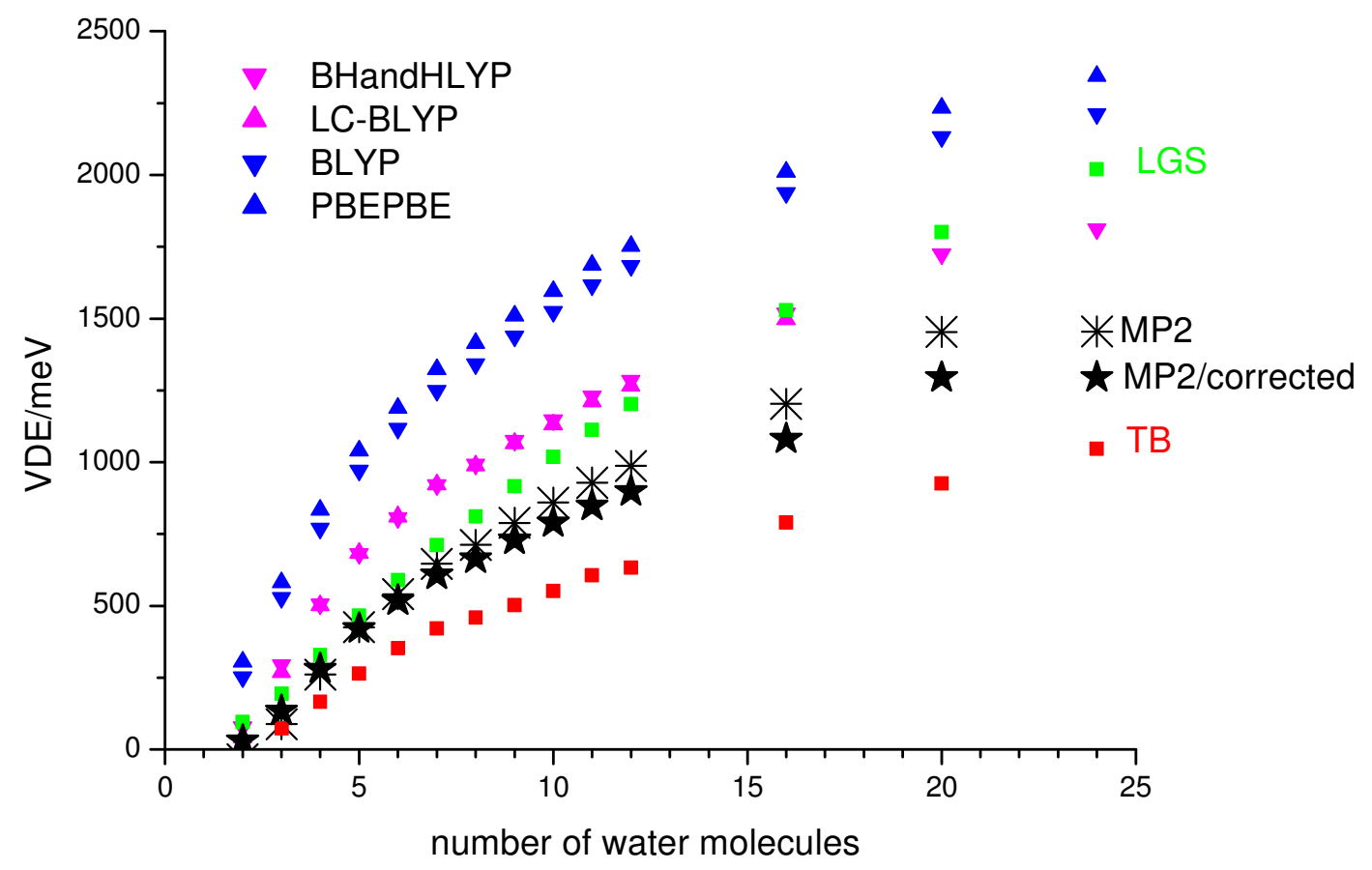


Figure 5.

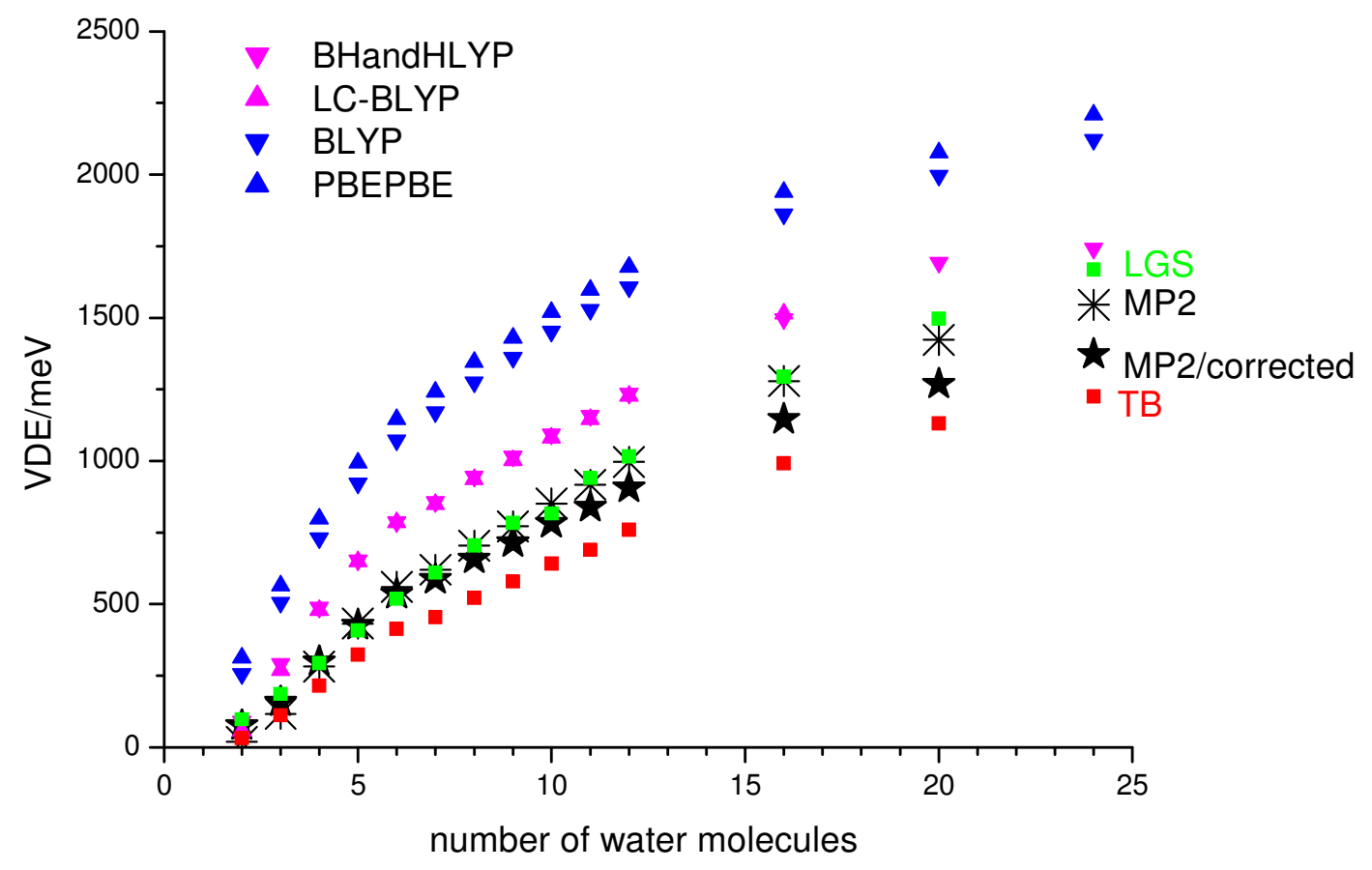


Figure 6.

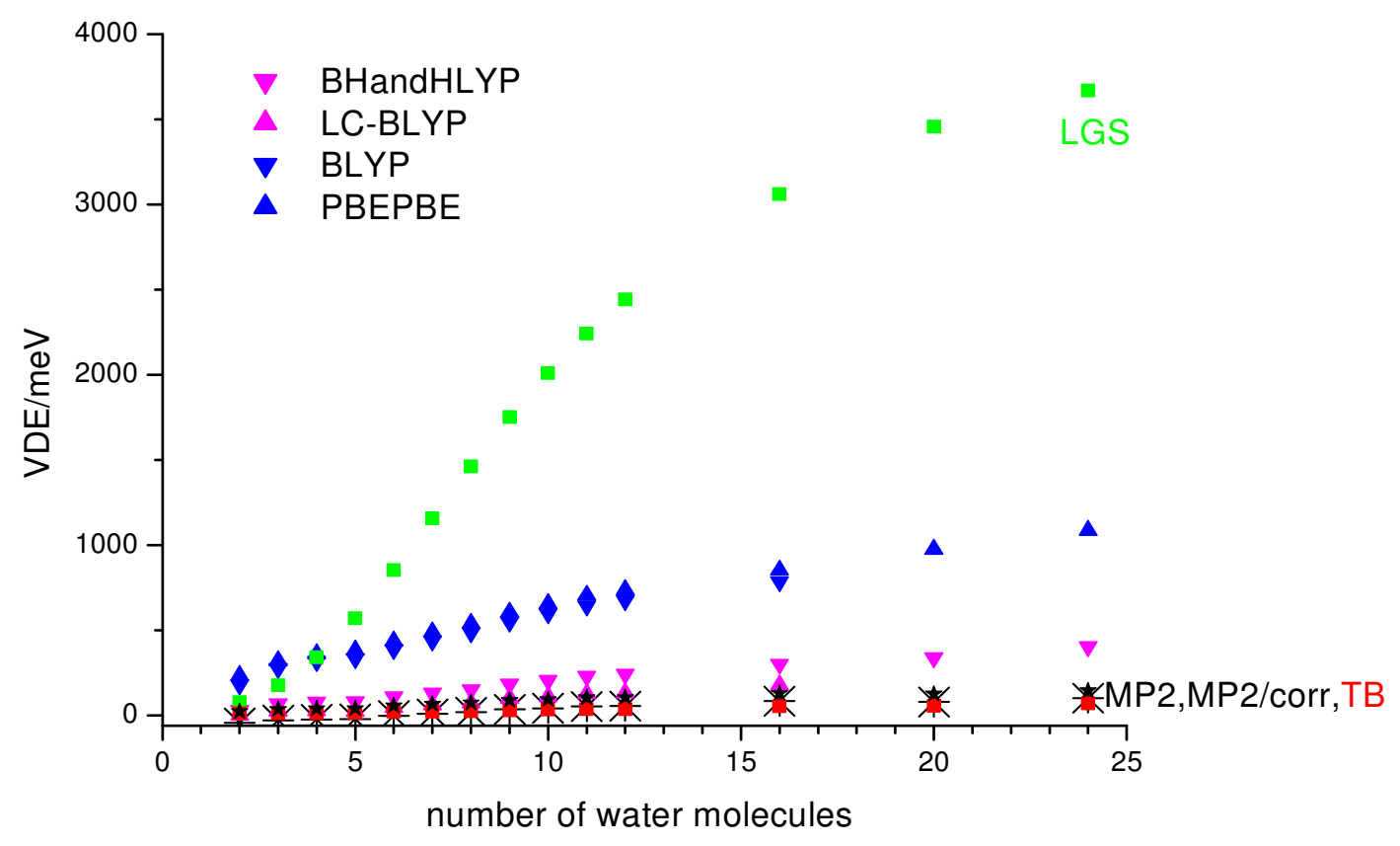


Figure 7.

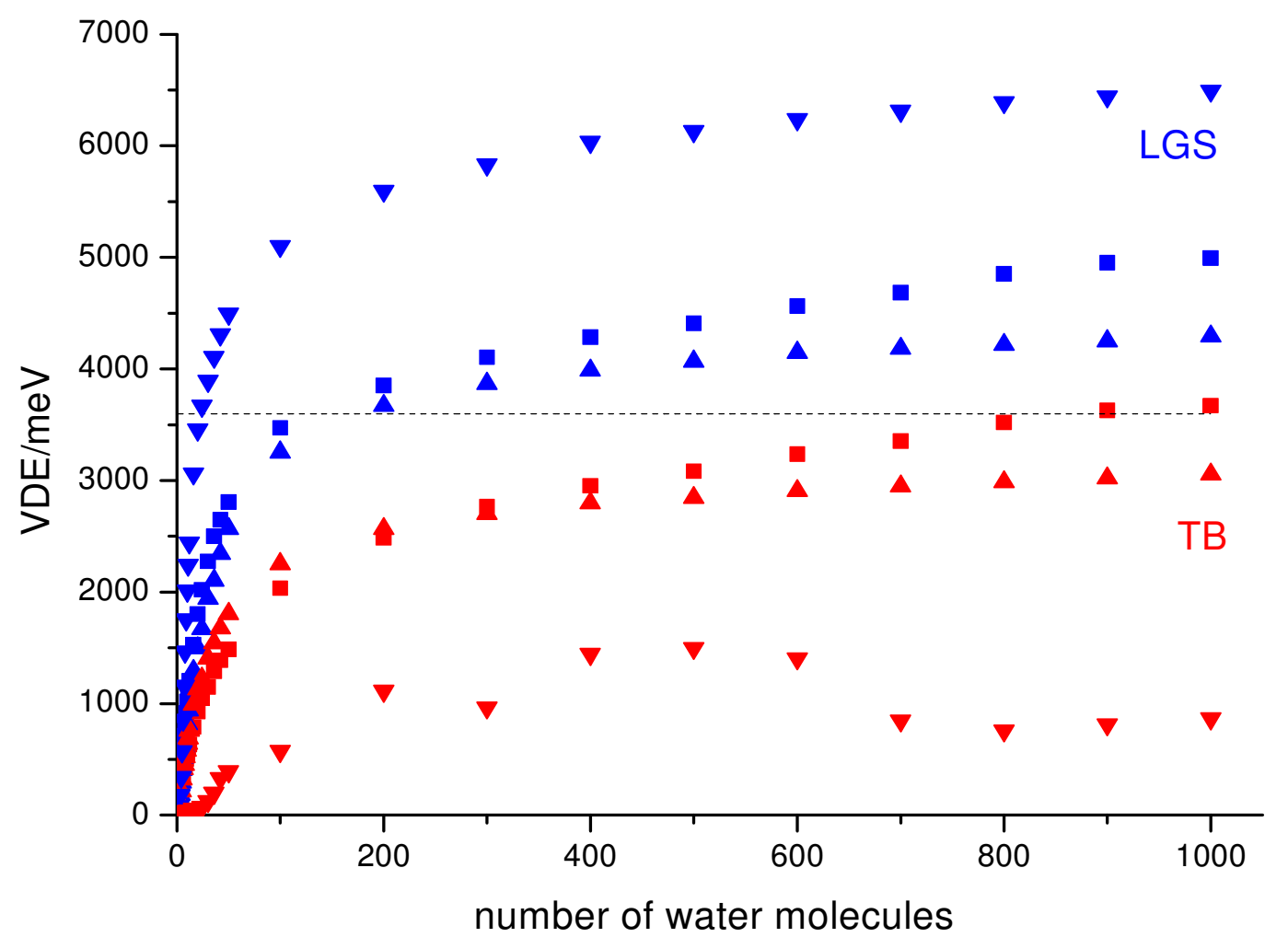


Figure 8.

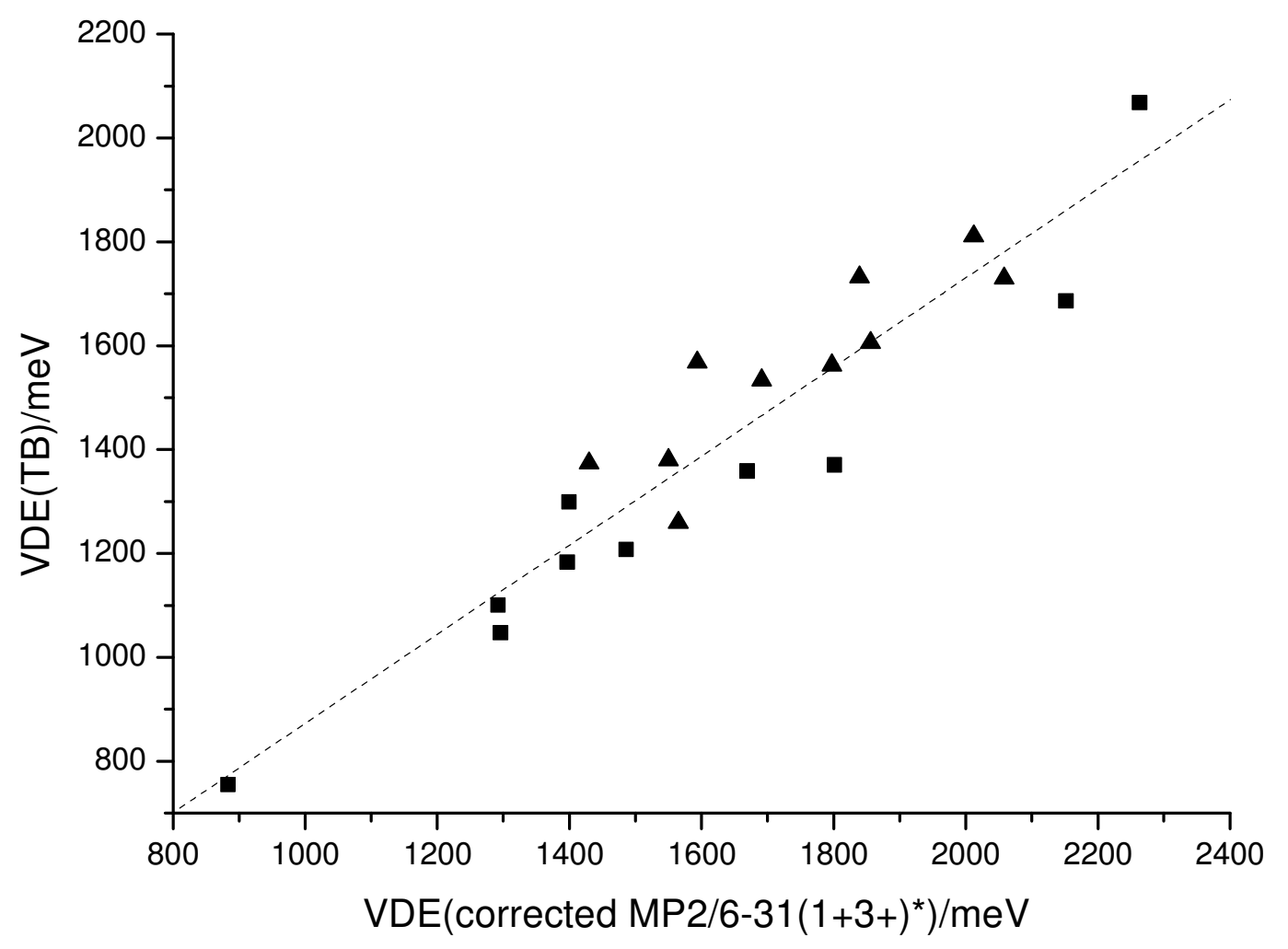




\section{Tables}

Table I. Calculated VDE values in meV for negatively charged water haxamer configurations taken from one-electron QCMD simulations. The results are averages of twenty configurations. The data in parentheses show the average deviation of the VDE (see Eq (1)) relative to calculations using the aug4-cc-pVDZ (first number) or the aug3-cc-pVTZ basis sets (second number).

\begin{tabular}{|c|c|c|c|}
\hline \multirow[t]{2}{*}{ Method } & \multicolumn{3}{|c|}{ Geometry } \\
\hline & LGS & TB interior & TB surface \\
\hline $\mathrm{MP} 2 / 6-31(1+3+) \mathrm{G}^{*}$ & $9.55(26.7,14.1)$ & $522(75.2,62.3)$ & $611(86.1,74.2)$ \\
\hline MP2/aug-cc-pVDZ & $-288(321,311)$ & $454(62.8,84.1)$ & $491(42.0,51.8)$ \\
\hline MP2/aug3-cc-pVDZ & $10.6(16.8,3.6)$ & $453(5.3,15.4)$ & $528(1.6,12.8)$ \\
\hline MP2/aug4-cc-pVDZ & 22.2 & 450 & 528 \\
\hline MP2/aug3-cc-pVTZ & 13.6 & 464 & 540 \\
\hline BHandHLYP/6-31(1+3+)G* & $127(-, 15.7)$ & $788(112,121)$ & $837(118,128)$ \\
\hline BHandHLYP/aug-cc-pVDZ & $-100(-, 220)$ & $637(45.9,37.4)$ & $671(42.1,45.3)$ \\
\hline BHandHLYP/aug3-cc-pVDZ & $119(-, 3.1)$ & $679(0.1,9.8)$ & $722(0.1,9.8)$ \\
\hline BHandHLYP /aug4-cc-pVDZ & - & 679 & 722 \\
\hline BHandHLYP /aug3-cc-pVTZ & 116 & 669 & 712 \\
\hline LC-BLYP/6-31(1+3+)G* & $66.3(21.7,21.5)$ & $794(137,144)$ & $844(139,145)$ \\
\hline LC-BLYP/aug-cc-pVDZ & $-216(288,278)$ & $625(40.9,35.6)$ & $660(52.5,46.6)$ \\
\hline LC-BLYP/aug3-cc-pVDZ & $53.0(10.8,2.8)$ & $659(0.1,6.7)$ & $709(0.1,6.6)$ \\
\hline LC-BLYP/aug4-cc-pVDZ & 60.7 & 659 & 709 \\
\hline
\end{tabular}




\section{References}

${ }^{1}$ L. Turi, and P. J. Rossky, Chem. Rev. 112, 5641 (2012).

${ }^{2}$ J. V. Coe, G. H. Lee, J. G. Eaton, S. T. Arnold, H. W. Sarkas, K. H. Bowen, C. Ludewigt, H. Haberland, and D. R. Worsnop, J. Chem. Phys. 92, 3980 (1990).

${ }^{3}$ J. R. R. Verlet, A. E. Bragg, A. Kammrath, O. Cheshnovsky, and D. M. Neumark, Science 307, $93(2005)$.

${ }^{4}$ L. Ma, K. Majer, F. Chirot, and B. von Issendorff, J. Chem. Phys. 131, 144303 (2009).

${ }^{5}$ F. Zappa, S. Denifl, I. Mähr, A. Bacher, O. Echt, T. D. Märk, and P. Scheier, J. Am. Chem. Soc. 130, 5573 (2008).

${ }^{6}$ G. B. Griffin, R. M. Young, O. T. Ehrler, and D. M. Neumark, J. Chem. Phys. 131, 194302 (2009).

${ }^{7}$ O. T. Ehrler, and D. M. Neumark, Acc. Chem. Res. 42, 769 (2009).

${ }^{8}$ R. N. Barnett, U. Landman, C. L. Cleveland, and J. Jortner, Phys. Rev. Lett. 59, 811 (1987).

${ }^{9}$ L. Turi, W.-S. Sheu, and P. J. Rossky, Science 309, 914 (2005).

${ }^{10}$ L. D. Jacobson, and J. M. Herbert, J. Am. Chem. Soc. 133, 19889 (2011).

${ }^{11}$ R. N. Barnett, R. Giniger, O. Cheshnovsky, and U. Landman, J. Phys. Chem. A. 115, 7378 (2011).

${ }^{12}$ R. E. Larsen, W. J. Glover, and B. J. Schwartz, Science 329, 65 (2010).

${ }^{13}$ J. R. Casey, A. Kahros, and B. J. Schwartz, J. Phys. Chem. B 117, 14173 (2013).

${ }^{14}$ R. A. Ogg, J. Am. Chem. Soc. 68, 155 (1946).

${ }^{15}$ A. Wallqvist, D. Thirumalai, and B. J. Berne, J. Chem. Phys. 86, 6404 (1987). 
${ }^{16}$ J. Schnitker, and P. J. Rossky, J. Chem. Phys. 86, 3462 (1987).

${ }^{17}$ R. N. Barnett, U. Landman, C. L. Cleveland, and J. Jortner, J. Chem. Phys. 88, 4421 (1988).

${ }^{18}$ A. Staib and D. Borgis, J. Chem. Phys. 103, 2642 (1995).

${ }^{19}$ L. Turi, and D. Borgis, J. Chem. Phys. 117, 6186 (2002).

${ }^{20}$ L. D. Jacobson, and J. M. Herbert, J. Chem. Phys. 133, 154506 (2010).

${ }^{21}$ V. K. Voora, J. Ding, T. Sommerfeld, and K. D. Jordan, J. Phys. Chem. B 117, 4365 (2013).

${ }^{22}$ A. Khan, J. Chem. Phys. 125, 024307 (2006).

${ }^{23}$ J. M. Herbert, and M. Head-Gordon, J. Phys. Chem. A 109, 5217 (2005).

${ }^{24}$ J. M. Herbert, and M. Head-Gordon, Phys. Chem. Chem. Phys. 8, 68 (2006).

${ }^{25}$ M. Boero, M. Parrinello, K. Terakura, T. Ikeshoji, and C. C. Liew, Phys. Rev. Lett. 90, 226403 (2003).

${ }^{26}$ T. Frigato, J. VandeVondele, B. Schmidt, C. Schütte, and P. Jungwirth, J. Phys. Chem. A 112, 6125 (2008).

${ }^{27}$ O. Marsalek, F. Uhlig, and P. Jungwirth, J. Phys. Chem. C 114, 20489 (2010).

${ }^{28}$ O. Marsalek, F. Uhlig, T. Frigato, B. Schmidt, and P. Jungwirth, Phys. Rev. Lett. 105, 043002 (2010).

${ }^{29}$ F. Uhlig, O. Marsalek, and P. Jungwirth, J. Phys. Chem. Lett. 3, 3071 (2012).

${ }^{30}$ F. Uhlig, O. Marsalek, and P. Jungwirth, J. Phys. Chem. Lett. 4, 338 (2013).

${ }^{31}$ F. Uhlig, J. M. Herbert, M. P. Coons, and P. Jungwirth, J. Phys. Chem. A 118, 7507 (2014).

${ }^{32}$ J. M. Herbert, Reviews in Computational Chemistry 28, ed. by A. L. Parrill and K. B. Lipkowitz, pp. 391-517 (2015). 
${ }^{33}$ V. P. Vysotskiy, L. S. Cederbaum, T. Sommerfeld, V. K. Voora, and K. D. Jordan, J. Chem. Theory Comput. 8, 893 (2012).

${ }^{34}$ M. Nooijen, and R.J. Bartlett, J. Chem. Phys. 102, 3629 (1995).

${ }^{35}$ L. Turi, J. Chem. Theory Comput. 11, 1745 (2015).

${ }^{36}$ Á. Madarász, P. J. Rossky, and L. Turi, J. Chem. Phys. 130, 124319 (2009).

37 Á. Madarász, P. J. Rossky, and L. Turi, J. Phys. Chem. A 114, 2331 (2010).

${ }^{38}$ T. Sommerfeld, A, DeFusco, and K. D. Jordan, J. Phys. Chem. A 112, 11021 (2008).

${ }^{39}$ L. D. Jacobson, C. F. Williams, and J. M. Herbert, J. Chem. Phys. 130, 124115 (2009).

${ }^{40}$ N. I. Hammer, J-W. Shin, J. M. Headrick, E. G. Diken, J. R. Roscioli, G. H. Weddle, and M. A. Johnson, Science 306, 675 (2004).

${ }^{41}$ N. I. Hammer, J. R. Roscioli, J. C. Bopp, J. M. Headrick, and M. A. Johnson, J. Chem. Phys. 123, 244311 (2006).

${ }^{42}$ F. A. Webster, P. J. Rossky and R. A. Friesner, Comput. Phys. Commun. 63, 494 (1991).

${ }^{43}$ L. Turi, J. Chem. Phys. 140, 204317 (2014).

${ }^{44}$ K. Toukan, and A. Rahman, Phys. Rev. B 31, 2643 (1985).

${ }^{45}$ L. Turi, M.-P. Gaigeot, N. Levy and D. Borgis, J. Chem. Phys. 114, 7805 (2001).

${ }^{46}$ M. A. Morrison and L. A. Collins, Phys. Rev. A 17, 918 (1978).

47 A. D. Becke, Phys. Rev. A 38, 3098 (1988). C. Lee, W. Yang, and R. G. Parr, Phys. Rev. B 37, 785 (1988). B. Miehlich, A. Savin, H. Stoll, and H. Preuss, Chem. Phys. Lett. 157, 200 (1989).

${ }^{48}$ H. Iikura, T. Tsuneda, T. Yanai, and K. Hirao, J. Chem. Phys. 115, 3540 (2001).

${ }^{49}$ A. D. Becke, J. Chem. Phys. 98, 1372 (1993). 
${ }^{50}$ J. P. Perdew, K. Burke, and M. Ernzerhof, Phys. Rev. Lett. 77, 3865 (1996). J. P. Perdew, K. Burke, and M. Ernzerhof, Phys. Rev. Lett. 78, 1396 (1997).

${ }^{51}$ C. Møller and M. S. Plesset, Phys. Rev. 46, 0618 (1934).

52 CFOUR, a quantum chemical program package written by J.F. Stanton, J. Gauss, M.E. Harding, P.G. Szalay with contributions from A.A. Auer, R.J. Bartlett, U. Benedikt, C. Berger, D.E. Bernholdt, Y.J. Bomble, L. Cheng, O. Christiansen, M. Heckert, O. Heun, C. Huber, T.-C. Jagau, D. Jonsson, J. Jusélius, K. Klein, W.J. Lauderdale, D.A. Matthews, T. Metzroth, L.A. Mück, D.P. O'Neill, D.R. Price, E. Prochnow, C. Puzzarini, K. Ruud, F. Schiffmann, W. Schwalbach, C. Simmons, S. Stopkowicz, A. Tajti, J. Vázquez, F. Wang, J.D. Watts and the integral packages MOLECULE (J. Almlöf and P.R. Taylor), PROPS (P.R. Taylor), ABACUS (T. Helgaker, H.J. Aa. Jensen, P. Jørgensen, and J. Olsen), and ECP routines by A. V. Mitin and C. van Wüllen. For the current version, see http://www.cfour.de.

${ }^{53}$ Gaussian 09, Revision B.01, M. J. Frisch, G. W. Trucks, H. B. Schlegel, G. E. Scuseria, M. A. Robb, J. R. Cheeseman, G. Scalmani, V. Barone, B. Mennucci, G. A. Petersson, H. Nakatsuji, M. Caricato, X. Li, H. P. Hratchian, A. F. Izmaylov, J. Bloino, G. Zheng, J. L. Sonnenberg, M. Hada, M. Ehara, K. Toyota, R. Fukuda, J. Hasegawa, M. Ishida, T. Nakajima, Y. Honda, O. Kitao, H. Nakai, T. Vreven, J. A. Montgomery, Jr., J. E. Peralta, F. Ogliaro, M. Bearpark, J. J. Heyd, E. Brothers, K. N. Kudin, V. N. Staroverov, R. Kobayashi, J. Normand, K. Raghavachari, A. Rendell, J. C. Burant, S. S. Iyengar, J. Tomasi, M. Cossi, N. Rega, J. M. Millam, M. Klene, J. E. Knox, J. B. Cross, V. Bakken, C. Adamo, J. Jaramillo, R. Gomperts, R. E. Stratmann, O. Yazyev, A. J. Austin, R. Cammi, C. Pomelli, J. W. Ochterski, R. L. Martin, K. Morokuma, V. G. 
Zakrzewski, G. A. Voth, P. Salvador, J. J. Dannenberg, S. Dapprich, A. D. Daniels, Ö. Farkas, J.

B. Foresman, J. V. Ortiz, J. Cioslowski, and D. J. Fox, Gaussian, Inc., Wallingford CT, 2009.

${ }^{54}$ A. T. Shreve, T. A. Yen, and D. M. Neumark, Chem. Phys. Lett. 493, 216 (2010).

${ }^{55}$ L. Turi, Á. Madarász, and P. J. Rossky, J. Chem. Phys. 125, 014308 (2006).

${ }^{56}$ J. V. Coe, S. M. Williams, and K. H. Bowen, Int. Rev. Phys. Chem. 27, 27 (2008).

${ }^{57}$ Y. Tang, H. Shen, K. Sekiguchi, N. Kurahashi, T. Mizuno, Y.-I. Suzuki, and T. Suzuki, Phys.

Chem. Chem. Phys. 12, 3653 (2010).

${ }^{58}$ K. R. Siefermann, Y. Liu, E. Lugovoy, O. Link, M. Faubel, U. Buck, B. Winter, and B. Abel, Nat. Chem. 2, 274 (2010).

${ }^{59}$ L. Turi, and Á. Madarász, Science, 331, 1387-c (2011).

${ }^{60}$ L. D. Jacobson, and J. M. Herbert, Science, 331, 1387-d (2011). 